ago and was found to have mitral stenosis and regurgitation with auricular fibrillation and a continuously irregular pulse. Since his discharge he has done two years' continuous work with one interruption of three months. His work involves standing for many hours a day and constantly running up and down the for many hours a day and constantly rumning up and down the cluring these two years and says that he is entirely dependent on the drug.

The following case of myocardial degeneration with auricular fibrillation responded well to digitalis, although the patient was a man of 70 years of age.

J. W., aged 70, metal smith, was atmitted into the Queen's Hospital on June 7th, 1911, suffering from shortness of breath, cough, and expectoration of gradually increasing severity for the past four years. On admission there was orthopnoea, cyanosis of the lips and ears, oedema of the legs, a lurge pulsatile liver, an enlarged spleen, and a rapid, small, continuously irregular pulse. Both ventricles were of large size, but the emphysematous condition of the lungs made it difficult to define the exact boundaries. There was a systolic murmur at the apex, audible in the axilla and at the tricuspid area. After twelve days' rest in bel, without drngs, the oedema had disappeared, but the pulse remained at about 90 per minute. A week later, after taking two granules of Nativelle's digitaline per day, the pulse had slowed to 60 per minute. Coincidently with this slowing the general condition of the patient greatly with this slowing the gens after his admission he greatly improved, and he left the had ceased, and the patient was able to mount stairs without any discomfort.

The following is a case of myocardial degeneration with auricular fibrillation in which the patient improved under digitalis in spite of the fact that he had cancer of the liver.

T. H., aged 56, bricklayer, was admitted into the Queen's Hospital on August 23rd, 1911, with carcinoma of the liver and a failing heart, evidenced by shortness of breath, cyanosis, oedema of the he of the legs and back, ascites, and a continuously irregular pulse of ventricles of the heart were enlarged, but emphysema made an exact deflnition impossible. There were no murmurs. Rest in the condition of the circulation, but six days later, after having taken 29 granule of digitaline, the pulse was 57 per minute, and of good volume all cyanosis and oedema had disappeared, and the ascites had diminished. A month later still, on 1 granule of digitaline per day, all ascites had disappeared, and the patient could mount stairs without any discomfort. This improvement in the heart's action has been maintained until the present time although the patient is losing strength and flesh as the result of malignant disease of the liver.

REFERENCES.

1 British Medical Jotrnal, October 14th and 21st, 1911. 2 Study of the Pulse. ${ }^{3}$ Diseases of the Heart. ${ }^{4}$ Lewis, Heart, vol. i, p. 311

\section{THE MANAGEMENT OF CARDIAC FAILURE IN DIPHTHERIA.}

By E. F. COGHLAN, M.B., C.M.Edin., M.R.C.S.Evg., L.R.C.P.LOND.

SOUTH-EASTERN FEVER HOSPITAI.

THE rational treatment of all forms of cardiac failure is to remove the cause or causes whenever possible; at the same time to considar the pathological changes in the cardio-vascular system with a view to amelioration of such changes; lastly, to consider the clinical aspect with a view to tiding the heart over the period of stress and preventing or relieving serious symptoms. With regard to the causal factor in diphtheria, it will generally be admitted that the action of the toxin on the heart muscle, resulting in certain degenerative changes, is the main canse of failure. The result of this action, though it can largely be prevented by the early, free administration of antitoxin, cannot be remedied once it has taken place. Unfortunately, in too many cases the antitoxin is not administered until the patient is admitted to hospital, often as late as the fourth or fifth day of the illness. The consequence is that valuable time is lost, and the main cause of cardiac failure may be beyond control. With reference to subsidiary causes, the early loss of appetite which so frequently occurs in diphtheria is a predisposing cause; whilst, on the other hand, the feeling of malaise probably exerts a beneficial influence, inasmuch as it necessitates rest, and so diminishes the heart work. That this point is of some importance seems evidenced by the fact that many patients who are conveyed by ambulance over considerable distances show on admission definite signs of cardiac depression, which pass awav after a night's rest.
There are, of course, many other factors tending to cause cardiac failure which may come into play in the case of adults suffering from diphtheria, but the main cause is the specific toxin, and it is largely under our control if adequate treatment is commenced early enough.

\section{Pathological Indications.}

Seeing that the changes which occur in the heart muscle are degenerative in nature and are so rapid, they furnish few indications for treatment. It is important to note, however, that in those cases characterized chiefly by failure of tonicity eliminative treatment by purgation and leeching is not only useless, but positively dangerous.

Clinical Indications.

Unfortunately we frequently see cases in which the causal factor has passed beyond our control, and we must rest content with expectant treatment. We therefore attempt to assist Nature in her efforts to promote resistancs sufficiently prolonged for the patient to throw off the disease, at the same time guarding against possible comp'i ations - thus, by the rational administration of food ald stimulant (if required), attention to sleep, and to the excreta, we endeavour to tide the patient over the crisis.

With reference to complications affecting the circulation, undoubtedly the most serious is that type of cardiac failure associated with persistent vomiting. It is so fatal and its early recognition so important that $I$ append a note on its differential diagnosis. When a patient suffering from severe diphtheria begins to vomit after the seventh day of the illness, we must remember that there are several possible explanations; thus, it may turn out that the dreaded cardiac vomiting has supervened, whilst, on the other hand, the vomiting may be due to the onset of scarlet fever. It is not possible to exclude scarlet fever with certainty at the commencement, as it sometimes begins with vomiting, associated with a normal or subnormal temperature. Howerer, a few hours generally makes the diagnosis clear. Measles may also commence with vomiting, and the fact that it is frequently afebrile in its very early stages and not infrequently commences without even coryza or suffusion of the conjunctiva may mislead one, unless a sharp look-out is kept for Koplik's spots.

Serum reaction may commence with an attack of vomiting and cardiac depression (especially the urticarial type of serum rash), but here again the lapse of a few hours generally clears up the diagnosis.

$$
\text { "Cardiac" Vomiting. }
$$

The following signs indicate the probability of myocardial involvement:

1. Certain Types of Faucial Attack.-When the faucial attack is severe and is complicated by involvement of the naso-pharynx and palate, the probability that the onset of vomiting may indicate commencing cardiac failure is considerable.

2. Rapidity of the Pulse.--It is generally recognized that in simple febrile conditions, for every rise in temperature of $1 \circ \mathrm{F}$. there is corresponding increase in the frequency of the pulse of 10 beats per minute. Whenever in diph. theria the pulse-rate increases out of proportion to the rise of temperature, involvement of the myocardium may be suspected. This sign, which was observed by Dr. Caiger many years ago, is one of the earliest and most reliable indications of threatened failure.

3. Auscultatory Signs.- The presence of the extra-systole with or without a consecutive intermission of the heart's action is also important evidence that the heart wall is involved in the morbid process. It carries with it a grave prognosis, when it first appears between the seventh and seventeenth days, but when it commences after the first three weeks its prognostic significance is nore favourable. In addition to the above, reduplication of the first sound, followed by accentuated second, is a sign of advanced myocardical disease, as is also marked approxi. mation. On the other hand, shortening of the first sound, accompanied by prolongation of the interval between it and the second sound, is an indication of less adyanced disease. Lastly, in some cases in which the function of contractility is depressed, the heart sounds may become almost inaudible. 
4. Anginoid pains occur, and are strong evidence of myocardial failure. Adult patients voluntarily complain of intermitting pains in the left arm, associated with a sense of constriction in the chest. Small children may suffer from pain during the stage of cardiac vomiting, and it is frequently referred to the abdomen, especially the epigastric region. Its cardiac origin may be suspected when it is relieved by hot applications to the precordial region.

5. Urine.-The amount secreted rapidly diminishes, and there may be complete suppression. Albumen may be present. It is commonly averred that albuminuria is an early and characteristic sign of diphtheria. Our experience, however, goes to prove that it is found in the more serious type of case only. It is consequently of less value as a diagnostic sign than is commonly stated.

6. The Liver is generally enlarged.

\section{Treatment.}

Treatment of patients suffering from cardiac failure accompanied by persistent vomiting should be conducted on the following lines:

1. Position of the Patient in Bed.-The head should be low to prevent cerebral anaemia, while the foot of the bed should be elevated. This may help retention of the rectal feeds.

2. Clothing.-Patients during the stage of cardiac depression become, restless and throw off the bedclothes. 'They should therefore be warmly clad, and for the same reason hot-water bottles should be placed in the bed.

3. Nourishment.-All-food by the mouth should be stopped and nutrient enemata administered. The rectum should be irrigated twice daily with warm boracic solution, otherwise irritation may be set up, and retention of nutrients become impossible.

Treatment of the Cardiac Depression.

This must be combated by the administration of medicines. Various drugs have been used for this purpose chief amonst them being strychnine, belladonna, and adrenalin. Since administering them in combination hypodermically we had four successful cases, though, of course, we have also had our failures. The formula used is as follows:

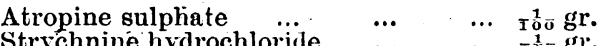

$$
\begin{aligned}
& \text { Strychnine hydrochloride } \ldots, 0 . \quad \ldots \quad \frac{1}{100} \text { gr. } \\
& \text { Adrenalin chloride solution }(1 \text { in } 1,000) \ldots \quad 5 \mathrm{~m}
\end{aligned}
$$

To be administere $\dddot{t}$ hypodermically every four hours.

That the above-mentioned combination exerciscd a beneficial influence on the favourable course the cases followed I am convinced, and in this opinion I am supported by Dr. Foord Caiger, who watched the cases with me from the very commencement.

Treatment of Urgent Symptoms.

Thirst.-This is first met by the administration twice daily of small enemata of normal saline solution, whilst teaspoonful doses of iced water may be allowed as soon as the vomiting ceases.

Dryncss of the Mouth may be alleviated by the application of such remedies as glycerine and boracic acid, hal strength.

Pain is most rapidly relieved by the frequent application of hot fomentations to the precordium.

Constipation.- It may be necessary to obtain an action of the bowels, and it is important to bear in mind that, owing to the serious cardiac depression, all purgatives are contraindicated. For this reason small enemata of glycerine and water are preferable.

Cases of Cardiac . Vomiting. Which recovered ender the Treatient Advocated.

$$
\text { CAS } \mathrm{I} \text {. }
$$

R. C. M., aged 8 years, was almitted to the South-Western Fever Hospital on October 26 th. He had been quite well on Fever Hospital on October 26th. He had bean quite well on sore throat; and on October 24 th swelling of the cervical glands, sore throat; and on October 24 th swelling of

On admission a deposit of thick opaque membrane comOn admission a deposit of thick opaque membrane com
pletely covered the inner surface of both tonsils and extended to the uvula. There was also general stvelling and oedema of the fauces. The cervical glands were much enlarged and rhinorrl o a was profuse. The heart apex beat in fifth interspace in ett nipple line. The heart sounds a little sharp and spaced. Pulse $114 ; 12,030$ units of antitoxin were injected and strychnine and brandy administered by mouth. October 27 th: The edges of membrane are beginning to curl up, phrsical signs remain unchanged, no albumen in urine. 8,000 units of antitoxin administered: October 28th: Membrane shows signs of disintegrating; cervical glands less swollen; physical signs in heart unchanged, but albumen one-eighth present in urine. Pulse 96 ; temperature normal. October 29th: Membrane peeling off. No change to note except that pulse is more rapid, 112. The patient progressed without material change until the evening of November 4 th, when he complained of not feeling so well. November 5th: Patient collapsed, inclined to vomit, general condition bad, surface cyanotic and extremities cold. The apex beat was well inside the left nipple line; the first sound showed the characteristic reduplication. Lower border of liver below the costal margin. Only passed $8 \mathrm{oz}$. of urine albumen present. Treatment as above. November 6th : Patient vomiting persistently. Tongue dry. Extremities cold. Patient vomiting persistently. Tongue dry. Extremities cold.
Hepatic enlargement remains. Pulse 130; temperature subHepatic enlargement remains. Pulse 130 ; temperature sub-
normal. November 7th, 8th, and 9th: Vomiting continuous. normal. November 7th, 8th, and 9th: Vomiting continuous. Pulse almost imperceptible at wrist. November 10th : Only
vomited once. Iiver enlargement less marked. November 11th: Vomiting ceased. Tongue moist and cleaning. Pulse 108, much stronger. November 12th: Pulse 96, force good, rhythm regular. Passed $14 \mathrm{oz}$. of urine. November 13th, 14th, and 15th: No recurrence of vomiting and patient able to take food by mouth in sufficient quantities; felt much stronger Urine increasing in quantity. November 16th: Complains of "pins and needles" in feet. During the next six weeks the patient suffered from paralysis of the muscles of accommodation, and ocular movements were paralysed, as also were the constrictors of the pharynx, adductors of the larynx and also the diaphragm. Eventually the patient made a good rend also the was able to leave his bed for a short time on December 28 th.

$$
\text { CASE II. }
$$

E. P. was admitted on February 27 th with the usual historr and a copious exudate on tonsils and uvula. On March 7 th she commenced to vomit, and showed the usual signs of cardiac depression, with oedema and dilatation. Rectal feeding was ordered, together with four-hourly injections of the solution of ordered, together with four-hourly injections of the solution of
atropin, strychnine, and adrenalin ; vomiting ceased after the
second day of treatment, but the signs of myocardial involvesecond day of treatment, but the signs of myocardial involve-
ment lasted for several weeks. Finally, after passing through a severe attack of post-diphtheritic paralysis, she eventually recovered.

CASE III.

W. L. G., aged 3 years and 2 months, was admitted on April 21st. The history was that he had vomited on April 12th, had sore throat on April 20th, and on April 21st swelling of cervical glands. On admission, there was injection of the fauces, an exudation covering either tonsil, and profuse rhinorrhoea; 12,000 units of antitoxin were administered, but the membrane continued to spread. ' On April-22nd vomiting com menced, and signs of cardiac failure, associated with a dilated left ventricle, appeared ; the patient continued very ill for some lays, showing most of the signs of cardiac depression above days, showing most of the signs of cardiac depression above enumerated. Eventually the vomiting ceased and the heart gained in strength, and the patient made a gradual but satis-
factory recovery. It is of interest to note that this patient factory recovery. It is of interest to note that this patient
suffered from several attacks of epistaxis, and several petechial spots appeared on the neck.

\section{CASE IV.}

A. T., aged 28 years, was admitted on July 3rd with the following history :-June 30 th: Headache and sore throat. July 2 nd Cervical glands enlarged. On admission examination revealed extensive exudation covering both tonsils, which were so extensive exudation covering both tonsils, which were so
swollen as to almost meet in the middle line. The exudate also extended to the uvula and soft palate. Submaxillary glands also extended to the uvula and soft palate. Submaxillary glands
much swollen on both sides. Heart sounds normal. Pulse 76, much swollen on both sides. Heart sounds normal. Pulse 76, colour good; 16,000 units of antitoxin were administered.
July 4th, 5th, and 6th: Exudation no less, otherwise not any change. July $7 \mathrm{th}$ : The patient feels better, the greater part of the exudate has separated. July 8 th, 9 th, and 10th: No change except that the patient had some difficulty in opening his mouth, in spite of the cervical glandular swelling having disappeared. July 11th: Persistent vomiting since early morning, patient collapsed. Heart sounds very weak, occasional extra systoles present. July 12th: Vomiting still. The apex beat was inside the left nipple line; the heart sounds weak; extra systoles followed by intermission. Some complaints of throbbing and feeling faint. Urine $13 \mathrm{oz}$., heavy deposit of albumen. July 13th: No more vomiting; pain in arm running down to July 13th: No more vomiting; pain in arm running down to of the chest; the pulse was intermittent; no extra systoles; of the chest; the pulse was intermittent; no extra systoles;
impulse no stronger. July 14th: Cardiac action stronger; no impulse no stronger. July 14th: Cardiac action stronger; no vomiting. Heart sounds rather spaced, albumen still present. July 16th: Complained of cardiac pain after an enema. No
signs of dilatation; no vomiting; heavy deposit of albumen. signs of dilatation; no vomiting; heavy deposit of albumen. July 17th : No more pain ; no vomiting; thick deposit of
albumen. July 19th: Heart action much stronger, but first sound reduplicated; albumen less. July 22nd: Heart action regular; no albumen; no bad symptoms. July 26th: No From this date cardiac action gradually became normal, but the patient did not escape some degree of paralysis.

The above case is one in which the cardiac affection was failure of contractility. It is of importance, as it indicates that recovery from such a grave condition need not be despaired of.

Dr. Caiger has kindly allowed me to use the notes of the above cases. 